\title{
Of the matrics of the equilibrium of the rod formation system based on the principle the duality of the problems of structures mechanics
}

\author{
Vladimir Monakhov ${ }^{1, *}$ \\ ${ }^{1}$ Penza State University of Architecture and Construction, Russia
}

\begin{abstract}
The formation of the equilibrium matrix of the core system in the matrix form is based on the use of the mechanical model of the system obtained by its discretization. The topological structure of the model is set using the graph and the accompanying incidence matrix. The matrix transformation of the vector of nodal displacements in combination with the extended incidence matrix allows determining the absolute elongations and distortions of each finite element. The composition of only two matrices (matrix of incidence and lengths of elements) and the skew vector leads to a geometric matrix characterizing the dependence of concentrated bending deformations in the calculated cross sections of the core system from the nodal displacements for a given load. Based on the duality principle, by transposing the geometric matrix, the equilibrium equation of the core system is derived in matrix form.
\end{abstract}

\section{Introduction}

By analyzing the stress state of the core system with the subsequent compilation of the equilibrium conditions of its sections, the equilibrium matrix can be generated in a static way [1]. In accordance with the principle of duality of efforts and deformations known in structural mechanics, the equilibrium matrix of the core system can also be obtained by transposing a geometric matrix. The construction of a geometric matrix is carried out on the basis of consideration of the schemes of possible displacements of the core system for a given external action, carried out manually [2]. The article developed an algorithm for constructing a geometric matrix of the core system and the formation of an equilibrium matrix in the automatic mode.

\section{The method of automatic formation of the equilibrium matrix}

The method of automatic formation of the equilibrium matrix is advisable to present it on a specific example of the core system, in particular, for the frame shown in Figure 1, a. Initially, the design scheme of the frame is subject to discretization: design sections (nodes)

* Corresponding author : monakhovnn@mail.ru 
and finite elements are outlined; the first are denoted by Arabic numerals $(i=1,2, \ldots, 10)$, the second - by Roman $(j=\mathrm{I}, \mathrm{II}, \ldots, \mathrm{VIII})$. At the same time, a global coordinate system $\eta \Omega \theta$ and local coordinate systems $x_{j} O_{j} y_{j}(\mathrm{j}=\mathrm{I}, \mathrm{II}, \ldots, \mathrm{VI})$ are introduced, the abscissa axes of which are combined with the axes of the elements (Fig. 1, b).
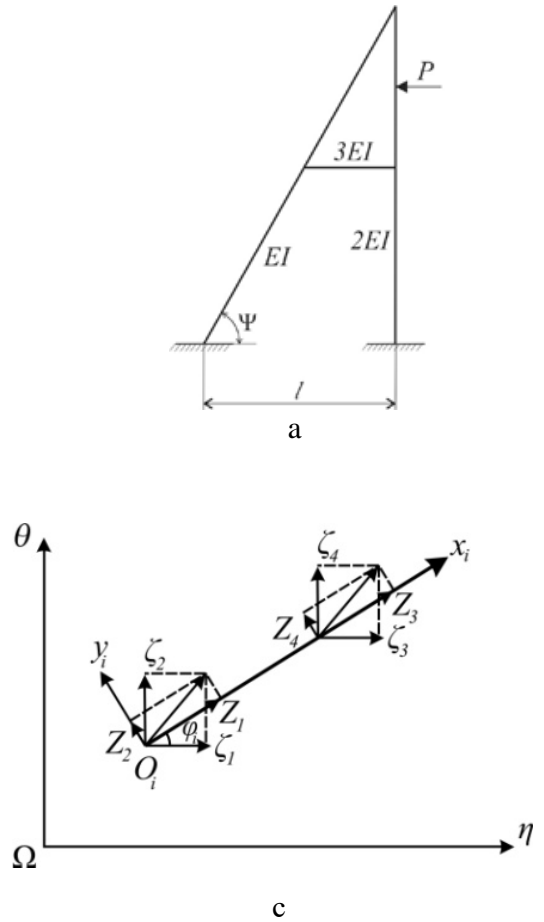

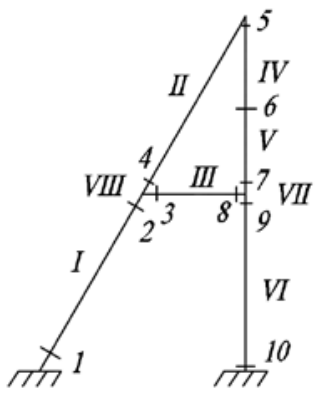

b

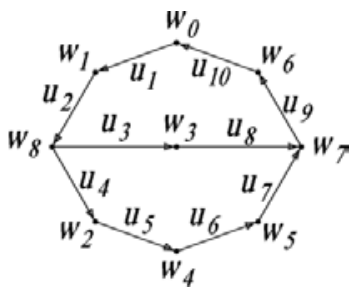

d

Fig. 1. The design scheme of the frame, its graph and the designation of the nodal displacements

To describe the structure of the frame design scheme, build a graph of a discrete frame model (Fig. 1, d), the vertices $w_{i}(i=1,2, \ldots, 8)$ of which are associated with finite elements, and arcs $u_{j}(j=1,2, \ldots, 10)$ - with nodes, and make up the incidence matrix accompanying it $[3,4]$

$$
[S]_{(6 \ltimes 8)}=\left[\begin{array}{cccccccc}
-1 & 1 & 0 & 0 & 0 & 0 & 0 & 0 \\
0 & 0 & 0 & -1 & 1 & 0 & 0 & 0 \\
0 & 0 & -1 & 0 & 0 & 0 & 0 & 1 \\
0 & 0 & 0 & 0 & -1 & 1 & 0 & 0 \\
0 & 0 & 0 & 0 & 0 & -1 & 1 & 0 \\
0 & 0 & 0 & 0 & 0 & 0 & 0 & 0
\end{array}\right] .
$$

Given the position of the local coordinate systems on the frame elements shown in Figure 1, c, d with the help of block rotation matrices 


$$
\left[\Psi_{i}\right]=\left[\begin{array}{ccc}
{\left[\psi_{i}\right]} & \vdots & 0 \\
\cdots & \vdots & \ldots \\
0 & \vdots & {\left[\psi_{i}\right]}
\end{array}\right] \quad(i=1,2, \ldots, 6)
$$

where

$$
\left[\psi_{i}\right]=\left[\begin{array}{rr}
\cos \psi_{i} & \sin \psi_{i} \\
-\sin \psi_{i} & \cos \psi_{i}
\end{array}\right] \quad(i=1,2, \ldots, 6)
$$

- the cell of rotation of the vector of displacements of one of the ends of the $i$ - th element, for each element it is easy to make the transition from global to local displacements $\bar{\zeta}$, according to the matrix transformation $\bar{Z}^{(i)}=\left[\Psi_{i}\right] \bar{\zeta}^{(i)} \quad(i=1,2, \ldots, 6)$. The set of similar transformations for all frame elements represented by a quasi-diagonal matrix of the form

$$
[\Psi]_{(24 \times 12)}=\left[\begin{array}{cccc}
{\left[\Psi_{1}\right]} & & & \\
& {\left[\Psi_{2}\right]} & & 0 \\
& {\left[\Psi_{3}\right]} & & \\
& & {\left[\Psi_{4}\right]} & \\
0 & & {\left[\Psi_{5}\right]} & \\
& & & {\left[\Psi_{6}\right]}
\end{array}\right] .
$$

where for example

$$
\begin{gathered}
{\left[\Psi_{1}\right]=\left[\Psi_{2}\right]=\left[\begin{array}{cccc}
\cos 60^{\circ} & \sin 60^{\circ} & 0 & 0 \\
-\sin 60^{\circ} & \cos 60^{\circ} & 0 & 0 \\
0 & 0 & \cos 60^{\circ} & \sin 60^{\circ} \\
0 & 0 & -\sin 60^{\circ} & \cos 60^{\circ}
\end{array}\right],} \\
{\left[\Psi_{4}\right]=\left[\Psi_{5}\right]=\left[\Psi_{6}\right]=\left[\begin{array}{cccc}
0 & -1 & 0 & 0 \\
1 & 0 & 0 & 0 \\
0 & 0 & 0 & -1 \\
0 & 0 & 1 & 0
\end{array}\right]}
\end{gathered}
$$

- matrix of rotation of the vector of nodal displacements for the first, second and other elements (for the horizontal it will be single), it allows to establish the relationship between local and global movements of the nodes of the entire frame in the matrix form.

In addition to the incidence matrix, a further developed incidence matrix $[\tilde{S}]$ of the graph should be formed, which is obtained on the basis of the matrix $[S]$ by its expansion. This procedure consists in transferring the significant elements of the matrix $[S]$ in the form of diagonal submatrices of the second order to the places corresponding to their degrees of freedom [5] 


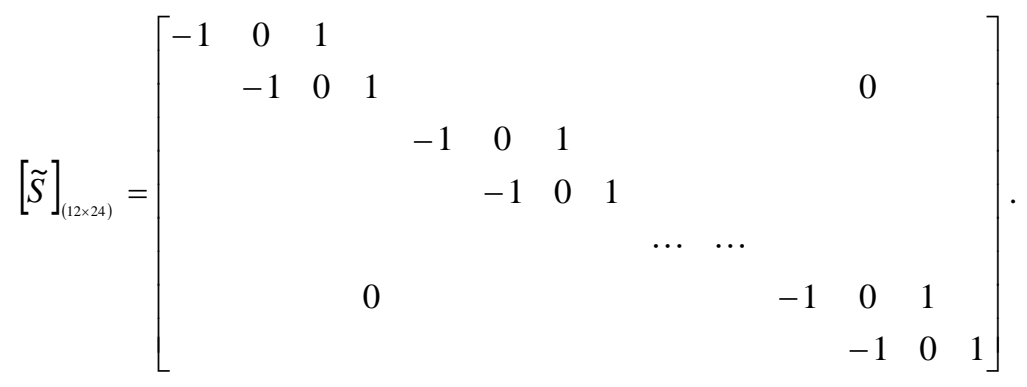

The dependence of displacement increments directly on displacements in the global system is characterized by transformation $\bar{\gamma}_{(12)}=[\Gamma]_{(12 \times 12)} \bar{\zeta}_{(12)}$, where $\bar{\gamma}_{(12)}=\left(\lambda_{1}, \chi_{1}, \lambda_{2}, \chi_{2}, \ldots, \lambda_{6}, \chi_{6}\right)$ is the column vector of the nodal displacements of the finite element displacements in the longitudinal direction $\lambda_{i}(i=1,2, \ldots, 6)$ and perpendicular to it $\chi_{i}(i=1,2, \ldots, 6)$. The transformation matrix $[\Gamma]$, which is found by the formula $[\Gamma]_{(12 \times 14)}=[\tilde{S}]_{(12 \times 24)}[\Psi]_{(24 \times 12)}$, is further corrected by crossing out the odd lines in it. Thus, in determining the internal forces of the frame, longitudinal deformations of finite elements are excluded. After deleting the specified rows come to the matrix

$$
[G]_{(6 \times 12)}=\left[\begin{array}{cccccccccccc}
-c & -s & -s & c & 0 & 0 & 0 & 0 & 0 & 0 & 0 & 0 \\
0 & 0 & s & -c & -s & c & 0 & 0 & 0 & 0 & 0 & 0 \\
0 & 0 & 0 & 1 & 0 & 0 & 0 & 0 & 0 & -1 & 0 & 0 \\
0 & 0 & 0 & 0 & -1 & 0 & 1 & 0 & 0 & 0 & 0 & 0 \\
0 & 0 & 0 & 0 & 0 & 0 & -1 & 0 & 1 & 0 & 0 & 0 \\
0 & 0 & 0 & 0 & 0 & 0 & 0 & 0 & -1 & 0 & -1 & 0
\end{array}\right] .
$$

Here, abbreviations for the functions of a given angle of inclination of the left frame frame are taken: $c=\cos 60^{\circ}, s=\sin 60^{\circ}$. This matrix allows you to find misalignments of the rods depending on the nodal displacements $\bar{\chi}_{(6)}=[G]_{(6 \times 12)} \bar{\zeta}_{(12)}$. Taking into account the immobility of the supports $\left(\zeta_{1}=\zeta_{2}=\zeta_{11}=\zeta_{12}=0\right)$, two extreme columns on each side should be omitted in the matrix $[G]_{(6 \times 12)}$; in addition, exclude the sixth, eighth and tenth columns, since the longitudinal deformations of the rods are negligible. Thus, a 6 by 5 rectangular matrix remains.

$$
\left[G_{0}\right]_{(6 \times 5)}=\left[\begin{array}{ccccc}
-s & c & 0 & 0 & 0 \\
s & -c & -s & 0 & 0 \\
0 & 1 & 0 & 0 & 0 \\
0 & 0 & -1 & 1 & 0 \\
0 & 0 & 0 & -1 & 1 \\
0 & 0 & 0 & 0 & -1
\end{array}\right]
$$


As a result of multiplying the transposed incidence matrix $[S]_{(8 \times 6)}^{T}$ by a matrix , $[L]_{(6 \times 6)}^{-1}$ $\left([L]_{(6 \times 6)}\right.$ - the KE length matrix), a matrix $[U]$ of dimension 8 by 6 is found. The geometric matrix with which the concentrated bending deformations in the nodes of the core system are determined according to $\overline{\boldsymbol{x}}_{(8)}=\left[H_{0}\right]_{(8 \times 5)} \bar{\zeta}_{(5)}$, is calculated by the formula $\left[H_{0}\right]_{(8 \times 5)}=[U]_{(8 \times 6)}\left[G_{0}\right]_{(6 \times 5)}$

$$
\left[H_{0}\right]_{(8 \times 5)}=\left[\begin{array}{ccccc}
0,87 & -0,5 & 0 & 0 & 0 \\
-0,87 & 0,5 & 0 & 0 & 0 \\
0 & 2 & 0 & 0 & 0 \\
-0,87 & 0,5 & 0,87 & 0 & 0 \\
0,87 & -0,5 & 1,44 & -2,31 & 0 \\
0 & 0 & -2,31 & 4,62 & -2,31 \\
0 & 0 & 0 & -2,31 & 2,31 \\
0 & -2 & 0 & 0 & 0
\end{array}\right] .
$$

The resulting matrix characterizes the deformation frame of the frame, resulting from nodal linear displacements. Since the frame bolt is adopted inextensible $\left(\zeta_{3}=\zeta_{9}\right)$, the last column of this matrix should be combined with the first and, at the same time, supplemented with two columns corresponding to two more possible deformation schemes. The latter are due to the rotation of the nodes at the corners $\vartheta_{5}, \vartheta_{6}$; their indices correspond to the general numbering of finite elements (Fig. 1, b).

If linear displacements of nodes are redesignated $\vartheta_{1}=\zeta_{3}, \vartheta_{2}=\zeta_{4}, \vartheta_{3}=\zeta_{5}, \vartheta_{4}=\zeta_{7}$, having accepted, and for angular ones, to continue numbering, i.e., count them $\vartheta_{5}, \vartheta_{6}$, then the concentrated bending deformations in the calculated cross sections of the frame can be calculated by the formula $\overline{\boldsymbol{x}}_{(10)}=[H]_{(10 \times 6)} \bar{\vartheta}_{6}$, where $\bar{\vartheta}=\left(\vartheta_{1}, \vartheta_{2}, \ldots, \vartheta_{6}\right)$ generalized vector of nodal displacements.

$$
[H]_{(10 \times 6)}=\left[\begin{array}{cccccc}
0,87 & -0,5 & 0 & 0 & 0 & 0 \\
-0,87 & 0,5 & 0 & 0 & 0 & -1 \\
0 & 2 & 0 & 0 & 0 & 1 \\
-0,87 & 0,5 & 0,87 & 0 & 0 & 1 \\
0,87 & -0,5 & 1,144 & -2,31 & 0 & 0 \\
-2,31 & 0 & -2,31 & 4,62 & 0 & 0 \\
2,31 & 0 & 0 & -2,31 & -1 & 0 \\
0 & -2 & 0 & 0 & -1 & 0 \\
1,16 & 0 & 0 & 0 & 1 & 0 \\
-1,16 & 0 & 0 & 0 & 0 & 0
\end{array}\right],
$$

Figure 2 shows the schemes of concentrated bending deformations: a) separately - with $\vartheta_{5}=1$, b) for the frame as a whole. 


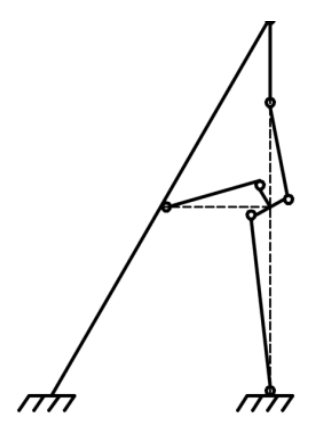

$\mathrm{a}$

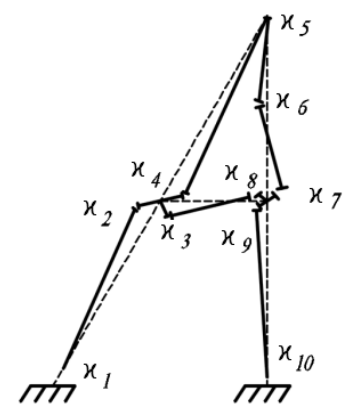

b

Fig. 2. Schemes of concentrated bending deformations of the frame:

a) deformation of the frame when turning the middle node at an angle $\vartheta_{5}=1$,

b) a complete scheme of concentrated flexural deformations

Using the principle of duality of efforts and deformations, by transposing a geometric matrix, a matrix is found

$$
[V]_{(6 \times 10)}=[H]_{(10 \times 6)}^{T}=\left[\begin{array}{cccccccccc}
0,87 & -0,87 & 0 & -0,87 & 0,87 & -2,31 & 2,31 & 0 & 1,16 & -1,16 \\
-0,5 & 0,5 & 2 & 0,5 & -0,5 & 0 & 0 & -2 & 0 & 0 \\
0 & 0 & 0 & 0,87 & 1,44 & -2,31 & 0 & 0 & 0 & 0 \\
0 & 0 & 0 & 0 & -2,31 & 4,62 & -2,31 & 0 & 0 & 0 \\
0 & 0 & 0 & 0 & 0 & 0 & -1 & -1 & 1 & 0 \\
0 & -1 & 1 & 1 & 0 & 0 & 0 & 0 & 0 & 0
\end{array}\right] .
$$

which as a result of multiplying by the vector the bending moments $\bar{M}_{(10)}$ in the cross sections of the frame and the subsequent addition with the load vector $\bar{P}=(0,0,0,-p, 0,0)$ leads to the equilibrium equation of the core system in the matrix form $[V]_{(10 \times 6)} \bar{M}_{(10)}+\bar{P}=0$.

The construction of the frame graph, the schemes of concentrated bending deformations, as well as the formation of the incidence matrices accompanying them and the rotation of the vector of nodal displacements together with the geometric matrix can be performed automatically [6, 7].

\section{Findings}

The method of automatic formation of a geometric matrix and an equilibrium matrix of the core system, proposed in the work, can be used to perform calculations of the core systems, both in terms of rigidity, and strength, stability and vibrations [8].

\section{Referenses}

1. A.F. Rzhanitsyn, Investigations in the Theory of Structures. Issue XXIV (1980).

2. A.M Protsenko, Theory of Ideally Elastoplastic Systems, "Science", Moscow, (1982)

3. A. Harari, Graph theory, URSS Euditorial, Moscow, (2003)

4. S.J. Fenves, F.H. Branin, Journal of structural division, vol. 89, ST4, (1963)

5. V. V. Reksha, Stroitel'naya mekhanika i raschet sooruzheniy, № 1 (1978) 
6. V.S. Zubov, Programmer's Handbook. Basic methods for solving graph problems and sorting, Izd. House "Filin", Moscow, (1999)

7. M. Swami, K. Tkhulasiraman, Graphs, networks, algorithms, "Mir", Moscow, (1984)

8. V.D. Potapov, Stroitel'naya mekhanika, T. 2. Стержневые системы, Moscow, "Stroisdat", (2007) 